\title{
The Right Ventricular Free Wall Strain and Functional Capacity Assessed by 6 Minutes Walk Test in Heart Faiure Patients with Reduced Ejection Fraction
}

\author{
Atakan Yanikoglu ${ }^{1}$, Refik Altekin ${ }^{2}$, and Aytul Belgi Yildirim ${ }^{2}$ \\ ${ }^{1}$ Antalya Private OFM Hospital \\ ${ }^{2}$ Akdeniz University Medical School
}

May 18, 2020

\begin{abstract}
Introduction The correlation of the right ventricular (RV) systolic function and functional capacity (FC) assessed by cardiopulmonary exercise testing (CPET) in HFrEF patients were demonstrated in previous studies In this study, we aimed to determine the correlation of RV free wall strain (RVfwlS), left ventricular (LV) strain parameters and other standart echocardiographic parameters with the FC assessed by the 6 minutes walk test $6 \mathrm{MWT}$ in HfrEF patients. Also, according to the calculated RVfwlS, the differences among HFrEF patients were examined. Method Dilated cardiomyopathy patients with an EF lower than $40 \%$ included in our study. After echocardiographic examinations, and venous blood sampling for NT-pro-BNP, the 6MWT performed for each participant by a physician blinded to echocardiographic examination. Results 50 patients with a mean EF of 28,78 $\pm 6,0 \%$ were included. The distance walked in 6MWT ( $6 \mathrm{MWT}$-dis) was significantly correlated with the NT-pro-BNP levels, E/Em-mean, left atrial volume index, RvfwlS, Sc (circumferential strain) and the Sl (longitudinal strain) . The Sc and the RVfwlS were found to be the independent predictors of the 6MWT-dis in multivariate analysis. According to mean RvfwlS which was $-18,50 \pm 4,83$, we grouped patients; Group 1 ( $\mathrm{n}=26)$ RvfwlS $>-18.5 \%$ and Group 2 ( $\mathrm{n}=24)$ RVfwlS $<-18.5 \%$. The Group 2 patients have higher NT-pro-BNP levels $(\mathrm{p}<0.01)$, lower 6MWT-dis $(\mathrm{p}<0.01)$, higher E/Em-mean $(\mathrm{p}<0.01)$ and lower $\mathrm{Sl}(\mathrm{p}<0.01)$. Conclusion The low RVfwlS in HFrEF patients could indicate cumulative exposure of RV to chronic increased LV filling pressures due to LV remodelling and dilatation.
\end{abstract}

\section{Method}

Dilated cardiomyopathy patients with an EF lower than $40 \%$ included in our study. After echocardiographic examinations, and venous blood sampling for NT-pro-BNP, the 6MWT performed for each participant by a physician blinded to echocardiographic examination.

\section{Results}

50 patients with a mean EF of $28,78 \pm 6,0 \%$ were included. The distance walked in 6MWT ( $6 \mathrm{MWT}$ dis) was significantly correlated with the NT-pro-BNP levels, E/Em-mean, left atrial volume index, RvfwlS, $\mathrm{S}_{\mathrm{c}}$ (circumferential strain) and the $\mathrm{S}_{1}$ (longitudinal strain). The Sc and the RVfwlS were found to be the independent predictors of the 6MWT-dis in multivariate analysis. According to mean RvfwlS which was $-18,50 \pm 4,83$, we grouped patients; Group $1(\mathrm{n}=26)$ RvfwlS $>-18.5 \%$ and Group $2(\mathrm{n}=24)$ RVfwlS $<$ $-18.5 \%$. The Group 2 patients have higher NT-pro-BNP levels $(\mathrm{p}<0.01)$, lower 6MWT-dis $(\mathrm{p}<0.01)$, higher E/Em-mean $(\mathrm{p}<0.01)$ and lower $\mathrm{S}_{\mathrm{l}}(\mathrm{p}<0.01)$.

\section{Conclusion}


The low RVfwlS in HFrEF patients could indicate cumulative exposure of RV to chronic increased LV filling pressures due to LV remodelling and dilatation.

\section{Introduction}

Right ventricular (RV) systolic dysfunction is common in heart failure (HF) and associated with poor prognosis. Chronic RV exposure to increased afterload due to impaired left ventricular (LV) filling and also chronic RV exposure to increased preload due to functional tricuspit insufficiency are the main factors of RV systolic dysfunction in HF patients with reduced ejection fraction (HFrEF) $(1,2)$. Prevalence of RV systolic function in HFrEF vary among studies $(3,4)$ In some studies, rather than the increased pulmonary pressures, the degree of LV systolic dysfunction was found to be correlated with the RV systolic function in these patients $(5,6)$.

The exact mechanisms of exercise intolerance in HF patients are not still completely understood. Pulmonary vascular congestion due to increased LV filling pressures and lower perfusion of skeletal muscles due to diminished cardiac output are the main pathophyisologic explanation for impaired functional capacity (FC) in HFrEF patients. Also abnormalities in peripheral vasodilatation response, metabolic changes in skeletal muscles, and changes in pulmonary alveolar-capillary bed are other explanatory mechanisms for reduced FC in HFrEF patients (7).

Previous studies could not determine any correlation of ejection fraction (EF) and FC in HFrEF patients (8). Also many studies adress the increased LV filling pressures for the major cause of FC limitation in these patients $(9,10)$ Although the RV systolic function could not be considered independently from LV systolic and diastolic abnormalities, the correlation of the RV systolic function and FC assessed by cardiopulmonary exercise testing (CPET) in HFrEF patients were demonstrated in previous studies $(11,12,13)$. 6 minutes walk test $(6 \mathrm{MWT})$ is a reliable method of evaluation of FC in HFrEF patients and correlates with the prognosis (14). Correlation of the distance walked in $6 \mathrm{MWT}$ with the peak oxygen consumption in CPET was shown in previous studies (15).

$2 \mathrm{~d}$ speckle tracking echocardiography (2DSTE) technique enables the assessment of myocardial strain and strain rate and it is an usefull tool to determine subtle myocardial changes. 2DSTE to calculate RV systolic function became a common tool in recent studies. RV strain is an objective marker of RV systolic function and the RV strain well correlates with calculated RV ejection fraction with cardiac magnetic resonance imaging (CMR) (16).

In this study, we aimed to determine the correlation of RV free wall strain (RVfwlS) and other standart echocardiographic parameters with FC assessed by the 6MWT in HfrEF patients. Also, according to the calculated RVfwlS, we aimed to determine the differences among HFrEF patients in terms of other echocardiographic parameters including 2DSTE assessment of LV systolic function, NT-pro-BNP levels and FC assessed by $6 \mathrm{MWT}$.

\section{Method}

Dilated cardiomyopathy patients whether ischemic or non-ischemic etiology with an EF lower than 40\% included in our study. Exclusion criteria were; acute decompensated HF and New York Heart Association (NYHA) class III and IV patients, inability to perform the 6MWT due to ocular, mental and orthopaedic problems, atrial fibrillation, mechanical or biologic valve replacement surgery, moderate and severe mitral and aortic stenosis and severe mitral, tricuspid and aortic insufficiency, end stage renal failure requiring dialysis, documented symptomatic obstructive chronic pulmonary disease and interstitial pulmonary disease, malignancy, cirrohosis, inadequate acoustic window. Study was approved by Akdeniz University Ethical Comission. Informed consents were taken from each patient. Each patients demographical, clinical data were gathered and echocardiographic examinations were performed and recorded to database.

Body mass indices and body surface areas by Mosteller formula calculated. Venous blood of each participant were sampled for NT-pro-BNP analysis and other laboratory data just before the $6 \mathrm{MWT}$. The Cockroft - 
Gault formula was used to estimate creatinine clearance rates. Elecsys NT-proBNP test (Roche Diagnostics,) was used to measure NT-pro-BNP levels.

GE - Vingmed Vivid 7 system (GE-Vingmed Ultrasound AS, Horten, Norway) echocardiography device was used for echocardiographic examinations. According to American Society of Echocardioraphy (ASE) 2D, Mmode, Doppler examinations were performed $(17,18,19)$. In addition, we used ratio of tricuspid regurgitant velocity (TRV) to RV outflow time-velocity integral (TVI-RVOT) for estimation of pulmonary vascular resistance, (20) 2D speckle tracking echocardiography analysis were performed with Echopac PC version 8 $\mathrm{GE}$, off-line from previous recordings according to recommendations of ASE and European Association of Cardiovascular Imaging (EACVI) (21). RV free wall strain (RVfwlS) were calculated from RV free wall views in apical four chamber according to the recommendations of ASE and EACVI (22).

The following echocardiographic parameters were examined : LAd: left atrium diameter, EF: ejection fraction calculated with bi-plane Simpson method, EDV: End diastolic volume calculated by bi-plane Simpson method, LAVI: left atrial volume index, RV-FAC \%: RV fractional area change, TAPSE: Tricuspid annular plane systolic excursion, Est-sPAP: estimated systolic pulmonary artery pressure, E/Em-mean : ratio of early diastolic mitral inflow velocity (E) to arithmetical mean of early diastolic lateral and septal tissue velocities (Em), TRV/TVI RVOT: ratio of tricuspid regurgitation velocity to the time velocity integral of the RV outflow tract systolic flow, $\mathrm{Sr}$ : radial strain, Sc : circumferential strain, Sl : longitudinal strain, RVfwlS: RV free wall longitudinal strain

After echocardiographic examination, 6MWT performed for each participant by a physician blinded to echocardiographic examination according to the guidelines and the distance (in meters) walked by each patient were recorded (23).

IBM SPSS Statistics for Windows, Version 23.0 (IBM Corp., Armonk, NY). was used for statistical analysis. In order to test for normality of distribution the Kolmogorov Smirnow test used. The Pearson test and Spearman tests were used for correlation analysis. In order to determine the effect of echocardiographic parameters to distance walked in 6MWT, the multivariable logistic regression analysis was used. According to the mean value of RVfwlS, patients divided into two groups (high RVfwlS group "Group 1" (RvfwlS > $-18.5 \%$ ) and low RVfwlS group "Group 2" (RVfwlS <-18.5\%). In order to determine difference among two groups ; for categorical variables, the chi square test, and for continous variables, the independent samples T-test and the Mann Whitney U tests were used.

\section{Results}

A total of 50 patients with a mean EF of $28,78 \pm 6,0 \%$ were included in our study . Demographic, clinical , laboratory and echocardiographic parameters were given in Table 1 and 2.

The distance walked in 6MWT ( 6MWT-dis) was significantly correlated with the NT-pro-BNP levels and RVfwlS. Also, strong correlation of 6MWT-dis with the LAVI , E/Em-mean, Est-sPAP , TRV/TVI RVOT, TAPSE, $\mathrm{S}_{\mathrm{c}}$ and the $\mathrm{S}_{1}$ was observed. The $\mathrm{S}_{\mathrm{r}}(\%)$, RV-FAC (\%) and BMI was also weakly correlated with the 6MWT-dis (Table 3).

Multivariate regression analysis among echocardiographic parameters, the $\mathrm{S}_{\mathrm{c}}$ and the RVfwlS were found to be the independent predictors of the 6MWT-dis ( Table 4).

Because of the mean RVfwlS was $-18,50 \pm 4,83 \%$ in our study, we sought appropriate to examine patients in two groups : Group $1(\mathrm{n}=26)$ the RvfwlS $>-18.5 \%$ and Group $2(\mathrm{n}=24)$ the RVfwlS $<-18.5 \%$. When we compare two groups; the Group 2 patients have higher NT-pro-BNP levels $(\mathrm{p}<0.01)$, lower 6MWT-dis $(\mathrm{p}<0.01)$, higher TRV/TVI RVOT $(\mathrm{p}<0.01)$, higher E/Em-mean $(\mathrm{p}<0.01)$, lower $\mathrm{Sl}(\mathrm{p}<0.01)$ and also lower TAPSE $(\mathrm{p}<0.01)$.

The Group 2 patients have lower EF ,higher EDV, higher LAVI and lower Sr, but there was a only a trend towards for statistical significance (Table 5). 
Table 1. Demographical and clinical parameters, and the 6MWT results of all study participitants

\begin{tabular}{ll}
\hline Age (years ): & $56.6 \pm 11.45$ \\
Female sex: & $9(18 \%)$ \\
Ischemic etiology: & $37(74 \%)$ \\
Diabetes Mellitus: & $15(30 \%)$ \\
BMI $\left(\mathrm{kg} / \mathrm{m}^{2}\right):$ & $26.2 \pm 4.74$ \\
Treatments used & \\
Beta Blocker : & $42(84 \%)$ \\
ACE-I or ARB: & $37(74 \%)$ \\
Spironolacton: & $20(40 \%)$ \\
Ivabradin : & $2(4 \%)$ \\
Statin : & $15(30 \%)$ \\
CRT: & $4(8 \%)$ \\
Laboratory data & \\
Hemoglobin (g/dl): & $13.5 \pm 1.5$ \\
NT-Pro-BNP (pg/ml): & $2876.3 \pm 3535.4,($ median : 1312.5) \\
GFR (ml/dk/1,73 m²)*: & $74.7 \pm 21.2$ \\
6MWT result & \\
6 MWT-Dis (meter) & $411.3 \pm 159.3$ \\
\hline
\end{tabular}

Abbreviations: BMI: Body mass index, ACE-I: Angiotensin converting enzyme ihnibitor, ARB: angiotensin receptor blocker, CRT: Cardiac resynchronization treatment, GFR: Glomerular filitration rate, 6MWT: 6 minutes walk test. $6 \mathrm{MWT}$-dis: The distance walked in 6 minutes walk test

* Calculated according to Cockroft formula.

Table 2. Echocardiographic parameters of all participants

\begin{tabular}{ll}
\hline Parameter & Mean \pm Std. Dev. \\
LAd (mm) & $45.26 \pm 4.19$ \\
EF $(\%)$ & $28.78 \pm 6.0$ \\
EDV $(\mathbf{m l})$ & $173,40 \pm 55,64$ \\
LAVI $\left(\mathbf{m l} / \mathbf{m}^{2}\right)$ & $45.59 \pm 18.43$ \\
RV-FAC (\%) & $41.89 \pm 11.61$ \\
TAPSE (mm) & $18.88 \pm 3.69$ \\
Est- sPAP (mmHg) & $47.6 \pm 19.3$ \\
E/Em-mean & $19.58 \pm 8.22$ \\
TRV/TVI RVOT & $0.18 \pm 0.09$ \\
S $_{\mathbf{r}}(\%)$ & $11.73 \pm 6.77$ \\
$\mathbf{S}_{\mathbf{c}} \mathbf{( \% )}$ & $-5.11 \pm 2.50$ \\
$\mathbf{S}_{\mathbf{l}}(\boldsymbol{\%})$ & $-7.35 \pm 2.90$ \\
RVfwlS (\%) & $-18.50 \pm 4.83$ \\
\hline
\end{tabular}

Abbreviations:Std.Dev.: standart deviation, LAd: left atrium diameter,. EF: ejection fraction (bi-plane Simpson method), EDV: End diastolic volume (bi-plane Simpson method), LAVI: left atrial volume index, RV-FAC: right ventricular fractional area change, TAPSE: Tricuspid annular plane systolic excursion, EstsPAP: estimated systolic pulmonary artery pressure, E/Em-mean : ratio of early diastolic mitral inflow velocity (E) to arithmetical mean of early diastolic lateral and septal tissue velocities (Em-mean), TRV/TVI RVOT: ratio of tricuspid regurgitation velocity to the time velocity integral of the right ventricular outflow 
tract systolic flow, $S_{r}$ : radial strain, $S_{c}$ : circumferential strain, $S_{1}$ : longitudinal strain, RVfwlS: right ventricular free wall longitudinal strain.

Table 3. The correlation between 6 MWT-Dis (meter) and clinical and echocardiographic parameters

\begin{tabular}{|c|c|}
\hline Parameter & 6 MWT-Dis (meter) \\
\hline Age & $-.120+$ \\
\hline $\operatorname{BMI}\left(\mathrm{kg} / \mathrm{m}^{2}\right)$ & $.306+^{*}$ \\
\hline Hemoglobin (g/dl) & $.249+$ \\
\hline $\operatorname{GFR}\left(\mathrm{ml} / \mathrm{dk} / 1.73 \mathrm{~m}^{2}\right)$ & $.214+$ \\
\hline NT-Pro-BNP (pg/ml) &,$- 699 ¥ * *$ \\
\hline LAd (mm) & $-.204 ¥$ \\
\hline EF (\%) & $.192+$ \\
\hline EDV $(\mathrm{ml})$ & $-.306+*$ \\
\hline LAVI $(\mathrm{ml} / \mathrm{m} 2)$ & $-.519+* *$ \\
\hline RV-FAC (\%) & $.343+*$ \\
\hline TAPSE (mm) & $.475 ¥ * *$ \\
\hline Est- sPAP (mmHg) & $-.413 ¥ * *$ \\
\hline TRV/TVI RVOT & $-.631 ¥ * *$ \\
\hline E/Em-mean & $-.555+* *$ \\
\hline $\mathrm{S}_{\mathrm{r}}(\%)$ & $.309+*$ \\
\hline$S_{c}(\%)$ & $-.444+* *$ \\
\hline$S_{1}(\%)$ & $-.443+* *$ \\
\hline RVfwlS (\%) & $-.574+* *$ \\
\hline
\end{tabular}

Abbreviations: 6MWT-dis: The distance walked in 6 minutes walk test, BMI: Body mass index, GFR: Glomerular filitration rate, LAd: left atrium diameter,. EF: ejection fraction (bi-plane Simpson method), EDV: End diastolic volume (bi-plane Simpson method), LAVI: left atrial volume index, RV-FAC: right ventricular fractional area change, TAPSE: Tricuspid annular plane systolic excursion, Est-sPAP: estimated systolic pulmonary artery pressure, E/Em-mean : ratio of early diastolic mitral inflow velocity (E) to arithmetical mean of early diastolic lateral and septal tissue velocities (Em-mean), TRV/TVI RVOT: ratio of tricuspid regurgitation velocity to the time velocity integral of the right ventricular outflow tract systolic flow, $\mathrm{S}_{\mathrm{r}}$ : radial strain, $\mathrm{S}_{\mathrm{c}}$ : circumferential strain, $\mathrm{S}_{\mathrm{l}}$ : longitudinal strain, RVfwlS: right ventricular free wall longitudinal strain.

+ Pearson correlation coefficient. ${ }^{*}$ Significant correlation $(\mathrm{p}<0,05)$

$¥$ Spearman rho correlation coefficient. ${ }^{* *}$ Significant correlation $(\mathrm{p}<0,01)$

Table 4. Multivariate regression analysis results of the correlated echocardiographic parameters in determination of $6 \mathrm{MWT}$-dis.

\begin{tabular}{lll}
\hline Parametre & $\beta$ & P value \\
E/Em-mean & -.250 & 0.082 \\
TRV/TVI RVOT & -.314 & 0.061 \\
$\mathbf{S}_{\mathbf{l}}(\%)$ & .121 & 0.380 \\
$\mathbf{S}_{\mathbf{c}}(\%)$ & -.263 & $0.028^{*}$ \\
RVfwlS (\%) & -.271 & $0.035^{*}$ \\
\hline
\end{tabular}

Adjusted $\mathrm{R}^{2}: 0,516$ 
$\mathrm{P}<0,001$

*statistical significance

Abbreviations: E/Em-mean : ratio of early diastolic mitral inflow velocity (E) to arithmetical mean of early diastolic lateral and septal tissue velocities (Em-mean), TRV/TVI RVOT: ratio of tricuspid regurgitation velocity to the time velocity integral of the right ventricular outflow tract systolic flow, $\mathrm{S}_{1}$ : longitudinal strain, $\mathrm{S}_{\mathrm{c}}$ : circumferential strain, RVfwlS: right ventricular free wall longitudinal strain.

Table 5. Comparision of participants clinical and echocardiographic parameters according to RVfwlS; Group 1 ( $>\mathbf{- 1 8 . 5 \% )}$ and Group $2(<-\mathbf{- 1 8 . 5 \%})$

\begin{tabular}{|c|c|c|c|}
\hline Parameter & $\begin{array}{l}\text { Group 1, }(\mathrm{n}=26) \\
\text { RVfwlS >-\%18.5 }\end{array}$ & $\begin{array}{l}\text { Group 2, }(\mathrm{n}=24) \\
\text { RVfwlS }<-\% 18.5\end{array}$ & $\mathbf{P}$ value \\
\hline Age & $54,8 \pm 9,3$ & $58,3 \pm 9,7$ & 0,196 \\
\hline Sex ( Female) & $6(\% 23,0)$ & $3(\% 12,5)$ & 0,467 \\
\hline BMI $\left(\mathrm{kg} / \mathrm{m}^{2}\right)$ & $26,3\left(25,25^{*}\right)$ & $25,8\left(25,77^{*}\right)$ & 0,900 \\
\hline Ischemic etiology & $19(\% 73,1)$ & $18(\% 75)$ & 1,0 \\
\hline Hemoglobin (g/dl) & $13,6 \pm 1,5$ & $13,5 \pm 1,5$ & 0,705 \\
\hline $\begin{array}{l}\text { GFR }(\mathrm{ml} / \mathrm{dk} / 1.73 \\
\left.\mathrm{m}^{2}\right)\end{array}$ & $74,6 \pm 19,5$ & $74,7 \pm 23,3$ & 0,984 \\
\hline NT-pro-BNP & $967,5\left(20,15^{*}\right)$ & $2724,0\left(31,29^{*}\right)$ & $<0,01$ \\
\hline 6 MWT-Dis (meter) & $488,2 \pm 130,9$ & $327,9 \pm 146,2$ & $<0,01$ \\
\hline EF $(\%)$ & $30,4 \pm 5,7$ & $27,0 \pm 6,0$ & 0,046 \\
\hline EDV $(\mathrm{ml})$ & $158,3 \pm 52,4$ & $189,7 \pm 55,3$ & 0,044 \\
\hline LAVI (ml/m2) & $41,3(21,92)^{*}$ & $52,1(29,38) *$ & 0,071 \\
\hline RV-FAC (\%) & $46,9 \pm 8,3$ & $36,4 \pm 12,3$ & 0,01 \\
\hline TAPSE (mm) & $20,6 \pm 2,8$ & $17,0 \pm 3,6$ & $<0,01$ \\
\hline Est- sPAP (mmHg) & $39,6(20,87)^{*}$ & $44,5(30,52)^{*}$ & 0,019 \\
\hline TRV/TVI RVOT & $0,147(18,58)^{*}$ & $0,255(33,0)^{*}$ & $<0,01$ \\
\hline E/Em-mean & $16,64 \pm 7,91$ & $22,76 \pm 7,46$ & $<0,01$ \\
\hline $\mathrm{S}_{\mathrm{r}}(\%)$ & $10,8(29,62)^{*}$ & $7,69(21,04)^{*}$ & 0,038 \\
\hline $\mathrm{S}_{\mathrm{c}}(\%)$ & $-5,15(23,08)^{*}$ & $-4,53(28,12)^{*}$ & 0,221 \\
\hline $\mathrm{S}_{1}(\%)$ & $-8,45 \pm 2,59$ & $-6,16 \pm 2,78$ & $<0,01$ \\
\hline
\end{tabular}

* Mann Whitney U test mean rank

Abbreviations: BMI: Body mass index, GFR: Glomerular filitration rate, 6MWT-dis: The distance walked in 6 minutes walk test, LAd: left atrium diameter,. EF: ejection fraction (bi-plane Simpson method), EDV: End diastolic volume (bi-plane Simpson method), LAVI: left atrial volume index, RV-FAC: right ventricular fractional area change, TAPSE: Tricuspid annular plane systolic excursion, Est-sPAP: estimated systolic pulmonary artery pressure, E/Em-mean : ratio of early diastolic mitral inflow velocity (E) to arithmetical mean of early diastolic lateral and septal tissue velocities (Em-mean), TRV/TVI RVOT: ratio of tricuspid regurgitation velocity to the time velocity integral of the right ventricular outflow tract systolic flow, $\mathrm{S}_{\mathrm{r}}$ : radial strain, $\mathrm{S}_{\mathrm{c}}$ : circumferential strain, $\mathrm{S}_{\mathrm{l}}$ : longitudinal strain, RVfwlS: right ventricular free wall longitudinal strain

\section{Discussion}

The $S_{c}$ and RwflS were found to be independent predictors of FC in HFrEF patients in our study. According to our study results, the patients with lower RVfwlS have greater echocardiographic increased LV filling pressure markers and NT-pro-BNP.

Previous studies seeking the question whether the echocardiography parameters are associated with the 
FC in HfrEF patients sign the elevated estimated LV filling pressures denoted by the increased E/Em ratios especially during exercise (24). Also in our study, the echocardiographic markers of elevated LV filling pressures and the NT-pro-BNP were also found to be correlated with the $6 \mathrm{MWT}$ results. As a similar finding in previous studies, both the LAVI and the Est- sPAP ( $\mathrm{mmHg}$ ) which are the markers of chronic exposure to increased LV filling pressures were found to be well correlated with the 6MWT results in our study(25). As a marker of pulmonary vascular resistance estimate the TRV/TVI RVOT ratio was also found to be well correlated with the 6MWT result. Reduced functional capacity and its association with the echocardiographic estimate of pulmonary vascular resistance and RV contractile reserve was well demonstrated in a previous study (26).

In our study the mean RVfwlS values in our study was concordant with the previous studies performed in HFrEF patients and the RVfwlS is well correlated with FC assessed by the 6MWT in HFrEF patients (27). Also in previous studies the RVfwlS was also found to be correlated with the FC assessed by the CPET $(13,28)$.

All of the three LV systolic strain parameters were found to be correlated with the FC assessed by the 6MWT. Previous studies demonstrated the correlation of the global longitudinal strain with the FC assessed by the CPET in HFrEF patients, however the association of the both $\mathrm{S}_{\mathrm{c}}$ and $\mathrm{S}_{\mathrm{r}}$ with the FC in these group of patients was not evaluated in these studies $(28,29)$.

The Sc and RVfwlS were found to be independent predictors in a best fit model to predict the FC assessed by $6 \mathrm{MWT}$. Further dilatation of the LV results with transverse orientation of basal and apical myocardium which are normally arranged in an oblique geometry(30). The lower $S_{c}$ in these group of patients may indicate the further systolic dysfunction due to further decrement of the systolic function of these transverse myocardial fibers. Also, in a study of Sveric et al. the torsion dysfunction and its association with the Sc was demonstrated in HFrEF patients (31).

The increased LV filling pressures resulting from further loss of LV twist / torsion was previously demonstrated (32). In our study we did not calculate the LV torsion, however the $\mathrm{S}_{\mathrm{c}}$ which is a systolic marker of the transversely oriented myocardial fibers which comprise for the LV twist / torsion mechanics was found to be an strong independent variable in our model to predict the FC assessed by 6MWT. In patients with reduced $\mathrm{S}_{\mathrm{c}}$, especially during exercise, the further LV filling pressure increase may limit the FC. The presence of greater LV filling pressure estimates in patients with the low RVfwlS group in our study results support this pathophysiologic explanation.

Despite the RVfwlS seems to be an independent predictor of FC assessed by the 6MWT in our study, the FC strongly correlated both with the hormonal (NT-pro-BNP) and echocardiographic (E/Em -mean and LAVI) markers of increased left LV filling pressures. Also, the patients with the lower RVfwlS have higher LV filling pressure markers.

We consider that the decrement of the RVfwlS eventuate as a result of cumulative exposure of RV to increased LV filling pressures. However the reason of why the RVfwlS rather than the E/Em mean ratio is an independent variable in our multivariate model may have two explanations. First, we do not have an echocardiographic information during exercise. The increased LV filling pressure is well documented in a previous study (26). The amount of LV filling pressure increase during exercise may have an greater impact. Second, the RVfwlS can be a long term monitoring marker of exposure to increased RV afterload.

Despite the $\mathrm{S}_{\mathrm{c}}$ determined as the strongest predictor of the FC assessed by 6MWT, the absence of statistical difference for the Sc among high and low RVfwlS patients can be explained by the our low study population.

According to our study results, HFrEF patients with lower RVfwlS exhibit patients who chronically exposed to increased resting RV afterload. As evidenced by the echocardiographic parameters in a previous study, the further increase of both the RV preload and afterload during exercise may also have an additional cumulative negative effect on the RV systolic functions (31).

Our results indicate that the RV systolic functions correlates with the LV remodelling process. As the LV 
becomes dilated, the oblique myocardial fibers become oriented in a more transverse fashion which in turn result with further increase of LV filling pressures due to decreased function of these fibers. The cumulative exposure of RV to increased LV filling pressures result with RV systolic dysfunction concurrent with the further FC impairment.

\section{Conclusion}

The RVfwlS and the $\mathrm{S}_{\mathrm{c}}$ in HfrEF patients were found to be strong independent predictors of the FC assessed by the $6 \mathrm{MWT}$ in our study. The low RVfwlS in HFrEF patients indicate cumulative exposure of RV to chronic increased LV filling pressures due to LV remodelling and dilatation. The conventional echocardiographic tools to evaluate RV functions may have limitations in HFrEF patients. The RV systolic functions of HFrEF patients can be better evaluated with 2DSTE method.

\section{Limitations of our study}

As well as our study lacks the information regarding the mitral insufficiency exacerbation during exercise, the absence of other exercise echocardiographic data also might have an influence on the analysis of the RV afterload impact on RV systolic function. Although the patients with poor acoustic window excluded from our study, speckle tracking echocardiography measurements may be affected by the thinned myocardium due to LV dilatation. Besides these limitations, the low study population may have an influence on our study results.

\section{References}

1. Kalogeropoulos AP, Vega JD, Smith AL, et al: Pulmonary Hypertension and Right Ventricular Function in Advanced Heart Failure. Congestive Heart Failure. 2011;17(4):189-198. doi:10.1111/j.17517133.2011.00234.x.

2. Ghio S, Gavazzi A, Campana C. Independent and additive prognostic value of right ventricular systolic function and pulmonary artery pressure in patients with chronic heart failure. ACC Current Journal Review. 2001;10(3):56. doi:10.1016/s1062-1458(01)00260-4.

3. Berkowitz R, Alhaj E, Manchikalapudi RB et al: Determinants of Right Ventricular Failure in Patients Admitted With Acute Left Heart Failure. Congestive Heart Failure. 2010;16(6):243-248. doi:10.1111/j.1751-7133.2010.00184.x.

4. Puwanant S, Priester TC, Mookadam F, et al: Right ventricular function in patients with preserved and reduced ejection fraction heart failure. European Journal of Echocardiography. 2009;10(6):733-737. doi:10.1093/ejechocard/jep052.

5. Gavazzi A, Ghio S, Scelsi L, et al. Response of the right ventricle to acute pulmonary vasodilation predicts the outcome in patients with advanced heart failure and pulmonary hypertension. American Heart Journal. 2003;145(2):310-316. doi:10.1067/mhj.2003.146

6. Desai RV, Meyer P, Ahmed MI, et al. Relationship between left and right ventricular ejection fractions in chronic advanced systolic heart failure: insights from the BEST trial. European Journal of Heart Failure. 2011;13(4):392-397. doi:10.1093/eurjhf/hfq206.

7. Pina Ileana L., Apstein CS, Balady GJ, et al. Exercise and Heart Failure. Circulation. 2003;107(8):1210-1225. doi:10.1161/01.cir.0000055013.92097.40.

8. Smart N, Haluska B, Leano R, Case C, Mottram PM, Marwick TH. Determinants of functional capacity in patients with chronic heart failure: Role of filling pressure and systolic and diastolic function. American Heart Journal. 2005;149(1):152-158. doi:10.1016/j.ahj.2004.06.017.

9. Terzi S, Sayar N, Bilsel T, et al. Tissue Doppler imaging adds incremental value in predicting exercise capacity in patients with congestive heart failure. Heart and Vessels. 2007;22(4):237-244. doi:10.1007/s00380-006-0961-x.

10. Hadano Y, Murata K, Yamamoto T, et al. Usefulness of Mitral Annular Velocity in Predicting Exercise Tolerance in Patients With Impaired Left Ventricular Systolic Function. The American Journal of Cardiology. 2006;97(7):1025-1028. doi:10.1016/j.amjcard.2005.10.044.

11. Nunes MDCP, Beloti FR, Lima MMO, et al. Functional capacity and right ventricular function 
in patients with Chagas heart disease. European Journal of Echocardiography. 2010;11(7):590-595. doi:10.1093/ejechocard/jeq022.

12. Zaborska B, Smarż K, Makowska E, et al. Echocardiographic predictors of exercise intolerance in patients with heart failure with severely reduced ejection fraction. Medicine. 2018;97(28). doi:10.1097/md.0000000000011523.

13. Sljivic A, Kleut MP, Bukumiric Z, Celic V. Association between right ventricle two- and threedimensional echocardiography and exercise capacity in patients with reduced left ventricular ejection fraction. Plos One. 2018;13(6). doi:10.1371/journal.pone.0199439.

14. Giannitsi S, Bougiakli M, Bechlioulis A, Kotsia A, Michalis LK, Naka KK. 6-minute walking test: a useful tool in the management of heart failure patients. Therapeutic Advances in Cardiovascular Disease. 2019;13:175394471987008. doi:10.1177/1753944719870084.

15. Rostagno C, Gensini GF. Six minute walk test: a simple and useful test to evaluate functional capacity in patients with heart failure. Internal and Emergency Medicine. 2008;3(3):205-212. doi:10.1007/s11739-008-0130-6.

16. Leong DP, Grover S, Molaee P, et al. Nonvolumetric Echocardiographic Indices of Right Ventricular Systolic Function: Validation with Cardiovascular Magnetic Resonance and Relationship with Functional Capacity. Echocardiography. 2011;29(4):455-463. doi:10.1111/j.1540-8175.2011.01594.x.

17. Lang RM, Bierig M, Devereux RB, et al. Recommendations for Chamber Quantification: A Report from the American Society of Echocardiography's Guidelines and Standards Committee and the Chamber Quantification Writing Group, Developed in Conjunction with the European Association of Echocardiography, a Branch of the European Society of Cardiology. Journal of the American Society of Echocardiography. 2005;18(12):1440-1463. doi:10.1016/j.echo.2005.10.005.

18. Nagueh SF, Appleton CP, Gillebert TC, et al. Recommendations for the Evaluation of Left Ventricular Diastolic Function by Echocardiography. Journal of the American Society of Echocardiography. 2009;22(2):107-133. doi:10.1016/j.echo.2008.11.023.

19. Rudski LG, Lai WW, Afilalo J, et al. Guidelines for the Echocardiographic Assessment of the Right Heart in Adults: A Report from the American Society of Echocardiography. Journal of the American Society of Echocardiography. 2010;23(7):685-713. doi:10.1016/j.echo.2010.05.010.

20. Abbas AE, Fortuin F, Schiller NB, Appleton CP, Moreno CA, Lester SJ. A simple method for noninvasive estimation of pulmonary vascular resistance. Journal of the American College of Cardiology. 2003;41(6):1021-1027. doi:10.1016/s0735-1097(02)02973-x.

21. Voigt J-U, Pedrizzetti G, Lysyansky P, et al. Definitions for a Common Standard for 2D Speckle Tracking Echocardiography: Consensus Document of the EACVI/ASE/Industry Task Force to Standardize Deformation Imaging. Journal of the American Society of Echocardiography. 2015;28(2):183-193. doi:10.1016/j.echo.2014.11.003.

22. Badano LP, Kolias TJ, Muraru D, et al. Standardization of left atrial, right ventricular, and right atrial deformation imaging using two-dimensional speckle tracking echocardiography: a consensus document of the EACVI/ASE/Industry Task Force to standardize deformation imaging. European Heart Journal - Cardiovascular Imaging. 2018;19(6):591-600. doi:10.1093/ehjci/jey042.

23. ATS Statement. American Journal of Respiratory and Critical Care Medicine. 2002;166(1):111-117. doi:10.1164/ajrccm.166.1.at1102.

24. Santoro C, Sorrentino R, Esposito R, et al. Cardiopulmonary exercise testing and echocardiographic exam: an useful interaction. Cardiovascular Ultrasound. 2019;17(1). doi:10.1186/s12947-019-0180-0.

25. Hasselberg NE, Haugaa KH, Sarvari SI, et al. Left ventricular global longitudinal strain is associated with exercise capacity in failing hearts with preserved and reduced ejection fraction. European Heart Journal - Cardiovascular Imaging. 2014;16(2):217-224. doi:10.1093/ehjci/jeu277.

26. Guazzi M, Villani S, Generati G, et al. Right Ventricular Contractile Reserve and Pulmonary Circulation Uncoupling During Exercise Challenge in Heart Failure. JACC: Heart Failure . 2016;4(8):625-635. doi:10.1016/j.jchf.2016.03.007.

27. Morris DA, Krisper M, Nakatani S, et al. Normal range and usefulness of right ventricular systolic strain to detect subtle right ventricular systolic abnormalities in patients with heart fail- 
ure: a multicentre study. European Heart Journal - Cardiovascular Imaging. 2016;18(2):212-223. doi:10.1093/ehjci/jew011.

28. Salerno G, D'Andrea A, Bossone E, et al. Association between right ventricular two-dimensional strain and exercise capacity in patients with either idiopathic or ischemic dilated cardiomyopathy. Journal of Cardiovascular Medicine. 2011;12(9):625-634. doi:10.2459/jcm.0b013e328349a268

29. Donal E, Coquerel N, Bodi S, et al. Importance of ventricular longitudinal function in chronic heart failure. European Journal of Echocardiography. 2011;12(8):619-627. doi:10.1093/ejechocard/jer089

30. Buckberg GD. Basic science review: The helix and the heart. The Journal of Thoracic and Cardiovascular Surgery. 2002;124(5):863-883. doi:10.1067/mtc.2002.122439

31. Sveric KM, Ulbrich S, Rady M, et al. Three-Dimensional Left Ventricular Torsion in Patients With Dilated Cardiomyopathy - A Marker of Disease Severity -. Circulation Journal. 2017;81(4):529-536. doi:10.1253/circj.cj-16-0965

32. Popescu BA, Beladan CC, Călin A, et al. Left ventricular remodelling and torsional dynamics in dilated cardiomyopathy: reversed apical rotation as a marker of disease severity. European Journal of Heart Failure. 2009;11(10):945-951. doi:10.1093/eurjhf/hfp124 